\title{
INSTRUMENTOS PARA AVALIAÇÃO DA ADESÃO DE PROFISSIONAIS DE SAÚDE À HIGIENIZAÇÃO DAS MÃOS: REVISÃO INTEGRATIVA
}

\section{INSTRUMENTS FOR EVALUATING THE ADHERENCE OF HEALTH PROFESSIONALS TO HAND HYGINE: INTEGRATIVE REVIEW}

\section{Luana Ramos Garcia ${ }^{1}$ * Larissa Sousa Oliva Brun ${ }^{2} *$ Priscila Brandão $^{3} *$ Maithê de Carvalho e Lemos Goulart ${ }^{4} *$ Fernanda Garcia Bezerra Góes $^{5} *$ Fernanda Maria Vieira Pereira-Ávila $^{6}$}

\section{RESUMO}

Objetivo: identificar na literatura científica instrumentos para avaliação da adesão de profissionais de saúde às boas práticas de higienização das mãos. Método: revisão integrativa da literatura, realizada nos recursos informacionais Literatura Latino-Americana e do Caribe em Ciências da Saúde, Literatura Internacional em Ciências da Saúde, Base de Dados de Enfermagem, Cumulative Index to Nursing and Allied Health Literature e Scientific Eletronic Library Online, no mês de março de 2021. Resultados: analisaram-se 30 artigos, dos quais 11 (36,6\%) utilizaram instrumentos validados. Houve destaque para 23 estudos (74\%) que utilizaram a observação não participante para avaliar a adesão às boas práticas de higienização. Conclusão: os achados apontaram a existência de instrumentos para avaliar a adesão às boas práticas de higienização das mãos. Porém, faz-se necessário o incentivo à aplicação de instrumentos validados, principalmente aqueles que se configurem em estratégia multimodal.

Palavras-chave: Desinfecção das Mãos; Contenção de Riscos Biológicos; Segurança do Paciente; Pessoal de Saúde.

\begin{abstract}
Objective: to identify, in the scientific literature, instruments for assessing health professionals' adherence to good hand hygiene practices. Method: integrative literature review, carried out in the information resources Latin American and Caribbean Literature in Health Sciences, International Literature in Health Sciences, Nursing Database, Cumulative Index to Nursing and Allied Health Literature and Scientific Electronic Library Online, in March 2021. Results: 30 articles were analyzed, of which $11(36,6 \%)$ used validated instruments. There were 23 studies $(74 \%)$ that used non-participant observation to assess adherence to good hygiene practices. Conclusion: the findings pointed to the existence of instruments to assess adherence to good hand hygiene practices. However, it is necessary to encourage the application of validated instruments, especially those that are configured in multimodal strategy.
\end{abstract}

Keywords: Hand Disinfection; Containment of Biohazards; Patient Safety; Health Personnel.

\footnotetext{
${ }^{1}$ Graduanda em Enfermagem pela Universidade Federal Fluminense. Membro integrante do Laboratório de Pesquisa, Práticas e Ensino Simulado em Enfermagem (LAPESE).ORCID: https://orcid.org/0000-0003-0538-7104. E-mail: Luana.rgarcia@ hotmail.com

${ }^{2}$ Graduanda em Enfermagem pela Universidade Federal Fluminense. Membro integrante do Laboratório de Pesquisa, Práticas e Ensino Simulado em Enfermagem (LAPESE). ORCID: https://orcid.org/0000-0001-7978-7878. E-mail: larissabrun@ gmail.com

${ }^{3}$ Graduanda em Enfermagem pela Universidade Federal Fluminense. Membro integrante do Laboratório de Pesquisa, Práticas e Ensino Simulado em Enfermagem (LAPESE). ORCID: https://orcid.org/0000-0002-2635-9121. E-mail: priscilabrandao@id.uff.br

${ }^{4}$ Enfermeira. Doutora em enfermagem e biociências. Professora Adjunta do Departamento de Enfermagem da Universidade Federal Fluminense - Rio das Ostras. ORCID: https://orcid.org/0000-0003-2764-5290. E-mail: maithegoulart@gmail.com

${ }^{5}$ Enfermeira. Doutora em enfermagem. Professora Adjunta do Departamento de Enfermagem de Rio das Ostras da Universidade Federal Fluminense - Rio das Ostras. ORCID: https://orcid.org/0000-0003-3894-3998. E-mail: ferbezerra@ gmail.com

${ }^{6}$ Enfermeira. Doutora em ciências. Chefe e Professora Adjunta do Departamento de Enfermagem de Rio das Ostras da Universidade Federal Fluminense - Rio das Ostras. ORCID: https://orcid.org/0000-0003-1060-6754. E-mail: fernanddamaria@ hotmail.com
} 


\section{INTRODUÇÃO}

As Infecções Relacionadas à Assistência à Saúde (IRAS) são complicações recorrentes na prática assistencial e retratam um problema real na prestação de cuidados, acometendo pacientes hospitalizados e sendo transmitidas, principalmente, pelas mãos dos profissionais de saúde. São responsáveis por altas taxas de morbimortalidade, prolongamento do tempo de internação e aumento da resistência a antimicrobianos, gerando incapacidades e aumento de custos institucionais ${ }^{(1)}$.

A higienização das mãos (HM) é um procedimento indispensável na prevenção de IRAS e, quando realizada adequadamente, é a forma mais simples e econômica para impedir a disseminação de agentes patogênicos. Embora seja uma medida primordial para a segurança do profissional e do paciente, tem se apresentado como uma tarefa difícil ${ }^{(2)}$.

As mãos são consideradas um dos principais meios de atuação dos profissionais na assistência em saúde, assim sendo existe uma dependência direta entre o cuidado seguro e a HM de forma habitual e correta $^{(3)}$. A Organização Mundial da Saúde (OMS) incentiva a adesão às boas práticas de HM envolvendo ações multimodais que ressaltem o primeiro desafio global para a segurança do paciente, intitulado "Cuidado Limpo é Cuidado Mais Seguro". Essa estratégia é composta por cinco componentes que englobam diversos aspectos relacionados à prática de HM, tornando-se uma ferramenta eficaz para redução das IRAS ${ }^{(4)}$.

Apesar de tal incentivo e da ciência dos profissionais sobre a importância da HM e os momentos em que a técnica deve ser realizada, existem discrepâncias entre a teoria e a prática, na medida em que a adesão permanece baixa em diferentes cenários ${ }^{(3,5)}$. Portanto, além dos fatores institucionais, outros três elementos são essenciais para a prática de HM: agente tópico com eficácia antimicrobiana, procedimento adequado ao utilizá-lo (com técnica adequada e no tempo preconizado) e adesão regular no seu uso ${ }^{(6)}$.

$\mathrm{O}$ uso de instrumentos validados para a avaliação da adesão à HM é uma estratégia interessante no que se refere à segurança do profissional e do paciente, uma vez que proporciona um diagnóstico situacional e subsídios para a tomada de decisão no sentido do fortalecimento da cultura organizacional de prevenção. Logo, torna-se necessário congregar em um único estudo as melhores evidências disponíveis sobre a temática para gerar visibilidade e favorecer a aplicação na prática clínica e na pesquisa dos instrumentos já disponíveis na literatura científica.

Assim, o presente estudo objetivou identificar, na literatura brasileira e internacional, instrumentos para avaliação da adesão de profissionais de saúde às boas práticas de higienização das mãos. 


\section{MÉTODO}

Revisão integrativa da literatura, que seguiu as etapas do método, com formulação da questão de pesquisa, busca e coleta de dados na literatura, categorização e análise crítica dos estudos incluídos na síntese qualitativa, interpretação dos resultados com discussão e, por fim, síntese do conhecimento e apresentação da revisão ${ }^{(7)}$.

A questão norteadora baseou-se na estratégia $\mathrm{PICO}^{(8)} \quad[\mathrm{P}$ (população): profissionais assistenciais da saúde; I (intervenção): adesão à prática de higienização das mãos; C (comparação): instrumentos utilizados para avaliação; $\mathrm{O}$ (resultados): uso de instrumentos validados]. Portanto, formulou-se a seguinte questão: "Existem instrumentos validados para avaliação da adesão de profissionais de saúde às boas práticas de higienização das mãos?”.

A busca da produção científica foi realizada em março de 2021, por meio da busca sistematizada nos seguintes recursos informacionais: Literatura Latino-Americana e do Caribe em Ciências da Saúde (LILACS), Literatura Internacional em Ciências da Saúde (MEDLINE), Cumulative Index to Nursing and Allied Health Literature (CINAHL) e Base de Dados de Enfermagem (BDENF) e, ainda, na Bilbioteca Scientific Electronic Library Online (SciELO).

Utilizaram-se descritores selecionados nos Descritores em Ciências da Saúde
(DeCS/BIREME) e Medical Subject

Headings (MeSH), sendo eles: "Desinfecção das Mãos”, "Contenção de Riscos Biológicos", "Segurança do Paciente" e "Pessoal de Saúde", em português, e "Hand Disinfection", "Containment of Biohazards", "Patient Safety" e "Health Personnel", em inglês. As buscas foram realizadas com os descritores associados entre si, com todas as combinações possíveis, utilizando o operador boleando "AND". A seleção das publicações ocorreu por meio da leitura crítica dos resumos e aplicação dos seguintes critérios de inclusão: publicações no formato de artigos científicos, encontrados na íntegra; nos idiomas português, inglês e espanhol; e com utilização de instrumento para avaliação da adesão às boas práticas de HM. Os critérios de exclusão foram publicações em duplicidade nas bases de dados, artigos de reflexão e editoriais, revisões de literatura e estudos não relacionados ao tema.

Para elaboração do fluxograma de seleção de estudos (Figura 1), utilizou-se a estratégia Preferred Reporting Items for Systematic Review and Meta-Analyses

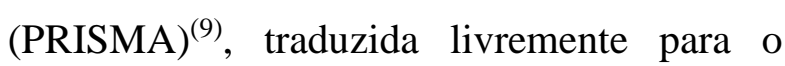
português. 
Figura 1 - Fluxograma da busca e seleção da literatura nas bases de dados. Rio da Ostras, RJ, Brasil, 2021
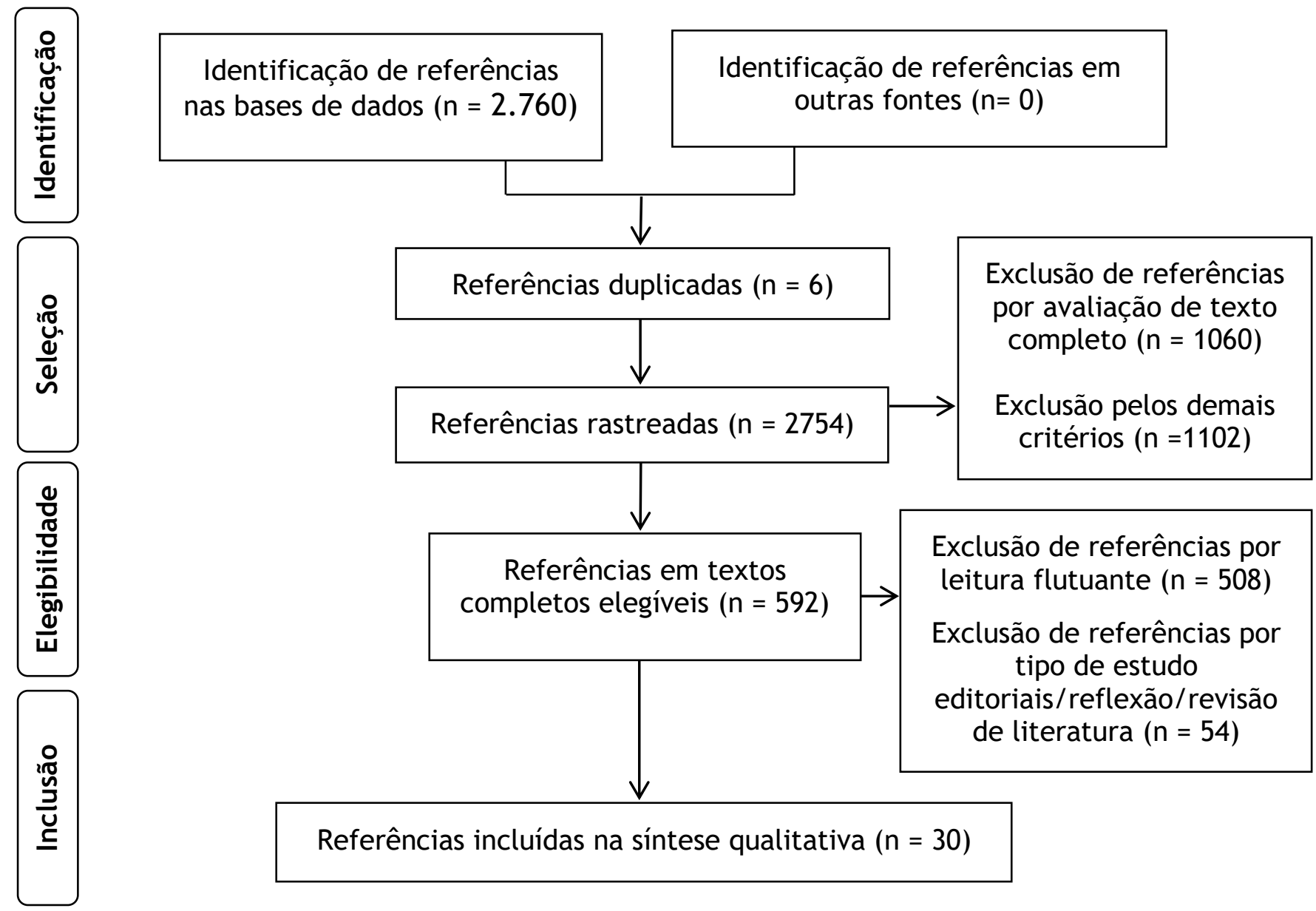

Fonte: dados de pesquisa.

Após a seleção dos artigos para leitura na íntegra, elaborou-se uma matriz de análise, proporcionando a síntese das evidências científicas. A matriz continha os seguintes dados: ordem; título; periódico; ano de publicação; webqualis; nível de evidência; objetivos; desenho do estudo; país; cenário; participantes; e instrumentos aplicados.

Para avaliar o nível de evidência, adotou-se a seguinte classificação: nível I - revisão sistemática ou meta-análise; nível II ensaio clínico randomizado controlado bem delineado; nível III - ensaios clínicos bem delineados sem randomização; nível IV estudos de coorte e de caso-controle; nível V - revisão sistemática de estudos descritivos e qualitativos; nível VI - um único estudo descritivo ou qualitativo; nível VII -opinião de autoridades e/ou relatório de comitês de especialistas $^{(10)}$. 


\section{RESULTADOS E DISCUSSÃO}

Analisaram-se 30 (100\%) artigos para leitura na íntegra e análise, sendo 6 na LILACS, 12 na MEDLINE, 7 na BDENF e 5 na CINAHL. Não foram selecionadas publicações na SciELO. Destaca-se que 1 $(3,3 \%)$ foi publicado em 2021, $2(6,6 \%)$ em 2020, 3 (10\%) em 2019 e $5(16,6 \%)$ no ano de 2018 e 19 (63,3\%) eram na língua inglesa.

Do total de artigos, $20(66,6 \%)^{(11-}$ $17,19,22,24,25,28-31,33,35,36,38,40)$ foram publicados em revistas multidisciplinares e 10
$(33,3 \%)^{(18,20,21,23,24,26,27,32,37,39)}$ em revistas científicas de Enfermagem. Em relação ao Qualis das revistas nas quais os artigos foram publicados, ressalta-se que $2(6,6 \%)^{(14,22)}$ foram em revistas de Qualis A1 e 9 $(30 \%)^{(16,19,25,28,31,33,39,40)}$ em Qualis A2.

No que se refere ao tipo de abordagem na pesquisa, $25(83,3 \%)$ publicações eram quantitativas, $\quad 21 \quad(70 \%)^{(11-13,15,17,18,20-24,26-}$ 28,30,32-35,38,40) artigos de cunho observacional e $28(93,3 \%)^{(11,12,14-21,23-37-40)}$ com nível de evidência IV (Figura 2).

Figura 2 - Caracterização dos artigos selecionados para análise segundo ordem, periódico, webqualis, ano e nível de evidência. Rio da Ostras, RJ, Brasil, 2021

\begin{tabular}{|c|c|c|c|}
\hline N. & Periódico/WebQualis & Ano & $\begin{array}{c}\text { Nível } \\
\text { de } \\
\text { evidência }\end{array}$ \\
\hline $\mathrm{A} 1^{(11)}$ & Am J Infect Control./B1 & 2009 & IV \\
\hline $\mathrm{A} 2^{(12)}$ & Rev paul pediatr. /B2 & 2009 & IV \\
\hline $\mathrm{A} 3^{(13)}$ & Am J Infect Control./B1 & 2010 & III \\
\hline $\mathrm{A} 4^{(14)}$ & Appl Nurs Res./A1 & 2010 & IV \\
\hline $\mathrm{A} 5^{(15)}$ & Am J Infect Control./B1 & 2011 & IV \\
\hline $\mathrm{A} 6^{(16)}$ & BMC Public Health/A2 & 2011 & IV \\
\hline $\mathrm{A} 7^{(17)}$ & Rev Calid Asist/B1 & 2011 & IV \\
\hline $\mathrm{A} 8^{(18)}$ & Rev Gaúcha Enferm/B1 & 2011 & IV \\
\hline $\mathrm{A} 9^{(19)}$ & J Hosp Infect/A2 & 2012 & IV \\
\hline $\mathrm{A} 10^{(20)}$ & Cienc. Enferm./B1 & 2013 & IV \\
\hline $\mathrm{A} 11^{(21)}$ & Esc Anna Nery/B1 & 2013 & IV \\
\hline $\mathrm{A} 12^{(22)}$ & Lancet Infect Dis/A1 & 2013 & III \\
\hline $\mathrm{A} 13^{(23)}$ & Rev Gaúcha Enferm/B1 & 2013 & IV \\
\hline $\mathrm{A} 14^{(24)}$ & Braz J Infect Dis/B3 & 2014 & IV \\
\hline $\mathrm{A} 15^{(25)}$ & Cad Saúde Pública/A2 & 2015 & IV \\
\hline $\mathrm{A} 16^{(26)}$ & Rev Gaúcha Enferm /B1 & 2015 & IV \\
\hline $\mathrm{A} 17^{(27)}$ & Rev iberoam Educ. investi. Enferm /B1 & 2016 & IV \\
\hline $\mathrm{A} 18^{(28)}$ & J Hosp Infect /A2 & 2017 & IV \\
\hline $\mathrm{A} 19^{(29)}$ & Rev Fund Care Online /B2 & 2017 & IV \\
\hline $\mathrm{A} 20^{(30)}$ & Ann Ig/B3 & 2018 & IV \\
\hline $\mathrm{A} 21^{(31)}$ & BMC Infect Dis /A2 & 2018 & IV \\
\hline $\mathrm{A} 22^{(32)}$ & $\mathrm{J}$ Nurs UFPE on line/B2 & 2018 & IV \\
\hline $\mathrm{A} 23^{(33)}$ & J Saúde Pública (Oxf)/A2 & 2018 & IV \\
\hline A2 $4^{(34)}$ & Rev enferm UFPE on line/B2 & 2018 & IV \\
\hline
\end{tabular}




\begin{tabular}{|c|c|c|c|}
\hline $\mathrm{A} 25^{(35)}$ & Infec Control Hosp Epidemiol/A2 & 2019 & IV \\
\hline $\mathrm{A} 26^{(36)}$ & Journal of Microbiology, Immunology and infection/A4 & 2019 & IV \\
\hline $\mathrm{A} 27^{(37)}$ & Rev enferm UFPE on line/B2 & 2019 & IV \\
\hline A2 $8^{(38)}$ & East Mediterr Health J/ s/qualis & 2020 & IV \\
\hline A29 $9^{(39)}$ & Rev. Bras. Enferm /A2 & 2020 & IV \\
\hline $\mathrm{A} 30^{(40)}$ & BMC Infect Dis /A2 & 2021 & IV \\
\hline
\end{tabular}

Fonte: os autores.

A HM é essencial na prática dos profissionais de saúde, contribuindo para prevenção das IRAS, no entanto é relevante que sejam realizadas orientações e treinamento acerca da temática ${ }^{(38)}$, incentivando, dessa forma, sua adesão.

A maioria dos estudos analisados foi realizada em ambiente hospitalar $(26 / 86,6 \%)^{(11-17,19-28,31,32-34,36,37-40)}, 1(3,3 \%)^{(18)}$ em unidade básica de saúde, $1(3,3 \%)^{(29)}$ em unidade de pronto atendimento, $1(3,3 \%)^{(30)}$ em casa de repouso e $1(3,3 \%)^{(35)}$ em instituto ortopédico. Ressalta-se que do total das pesquisas realizadas em hospitais, 13 $(43,3 \%)^{(13,15-17,19,21,22,24,25,27,33,36,37)}$ contaram com coleta de dados em todos os setores da instituição, $2(6,6 \%)^{(14,34)} \mathrm{em}$ oncologia, 5 $(16,6 \%)^{(23,26,32,39,40)}$ em unidades de terapia intensiva (UTI), $1(3,3 \%)^{(12)}$ em unidade de terapia intensiva neonatal (UTIN), 1 $(3,3 \%)^{(20)}$ em unidade de internação pediátrica, $1(3,3 \%)^{(11)}$ em unidade de oftalmologia, cardiologia, geriatria, doenças infecciosas e departamento de emergência, 1 $(3,3 \%)^{(38)}$ em setor de emergência hospitalar, $1(3,3 \%)^{(28)}$ em enfermaria de doenças infecciosas e apenas $1(3,3 \%)^{(31)}$ em UTIN, com berçário, suíte de parto e enfermaria cirúrgica. Contudo, não foram identificados setores hospitalares específicos como objeto frequente de investigação. Porém, dada as características do setor e quantidade de procedimentos invasivos realizados, um estudo baseado na UTI de um hospital universitário obteve um índice baixo de adesão à HM, inferior a 50\%, e tendência a níveis elevados de infecção ${ }^{(39)}$.

A equipe multidisciplinar de saúde foi predominante em 23 artigos $(76,6 \%)^{(13-15,17-}$ $19,22-28,30-36,38-40), \quad 3(10 \%)^{(11,21,29)}$ contaram exclusivamente com a equipe médica e de enfermagem, $1(3,3 \%)^{(20)}$ somente com a enfermagem, $2(6,6 \%)^{(12,16)}$ direcionados à HM dos pais/cuidadores e dos profissionais de saúde e apenas $1 \quad(3,3 \%)^{(37)}$ incluía profissionais de saúde, com o pessoal da limpeza, recepcionistas e telemarketing. A baixa adesão dos profissionais de saúde às boas práticas de HM foi apontada em 15 $(50 \%)^{(13,14,17,18,20,21,23-27,29,32-34)}$ dos estudos analisados.

Entretanto, apontaram-se médicos e auxiliares de enfermagem $^{(23)}$ e as equipes de enfermeiros ${ }^{(39)}$, médico e fisioterapeutas $^{(18,27,29,34)}$ como aqueles que demonstraram melhor execução e certa 
aderência à técnica correta, em relação aos demais profissionais de saúde. Evidenciou-se em dois artigos menor adesão nas indicações "antes do contato com o paciente" e "antes de procedimento asséptico", demonstrando-se, assim, ser o ponto de maior fragilidade de HM na assistência ${ }^{(32,37)}$ e a existência de lacunas de conhecimento referente à temática, acarretando na baixa adesão ${ }^{(26)}$. Enquanto em $u^{(39)}$ artigo a equipe de enfermagem esteve mais associada à $\mathrm{HM}$, sendo o momento "após o contato com o paciente" a ação mais presente.

Embora um dos $\operatorname{artigos}^{(27)}$ analisados tenha apontado que as equipes médicas e os enfermeiros possuem maior aderência à técnica correta de HM, em relação aos demais profissionais, outros estudos mencionam que são os fisioterapeutas e enfermeiros ${ }^{(6,32,33,39)}$. Cabe destacar que, corroborando os dados de baixa adesão, o padrão da técnica realizada pelos profissionais de saúde ainda é ineficaz, comprometendo a saúde dos pacientes e das equipes $^{(6,43-45)}$.

A aplicação de instrumentos de avaliação pode contribuir para o estabelecimento de metas e estratégias gerenciais e, também, educativas adequadas à realidade local. Diversos instrumentos foram utilizados para avaliar a adesão de profissionais à HM, porém dos artigos analisados $11(36,6 \%)^{(12,13,20,21,24,25,27,30,34,38,39)}$ utilizaram instrumentos validados, sendo eles:
$6(20 \%)^{(21,24,25,30,38,39)}$ previamente validados pela OMS, $2(6,6)^{(27,34)}$ validados por especialistas, $1(3,3 \%)^{(20)}$ validado por teste piloto e $2(6,6 \%)^{(12,13)}$ referem ter validado e testado o instrumento previamente, sem maiores detalhes.

Ressalta-se que os instrumentos validados e adaptados, em sua maioria, eram de autoria da OMS, destacando-se: "Guidelines on hand hygiene in health care"(23), "Guide to Implementation - A Guide to the implementation of the WHO Multimodal Hand Hygiene Improvement Strategy"(20-22,39), "My Five Moments For Hand Hygiene"(25,26,28,33,38) "Hand Hygiene Knowledge Questionnaire for Health-Care Workers"(25), "Perception Survey for HealthCare Workers of the WHO Clean your hands Campaign"(16), "Hand Hygiene Observation Form ${ }^{(24)}$. Um único artigo teve seus instrumentos baseados e adaptados de normas e diretrizes da Agência Nacional de Vigilância Sanitária (ANVISA) ${ }^{(32)}$.

Houve destaque para 23 estudos $(74 \%)^{(11-13,15,17,18,20-24,26-28,30,32-35,37-40)} \quad$ que utilizaram a observação não participante para avaliar a adesão às boas práticas de higienização (técnica e/ou momento em que ocorreu a higienização). Destes, $15^{(11-}$ 13,18,20,24,26,28,32-35,38-40) utilizaram somente o instrumento para observação. Os outros instrumentos aplicados foram quatro questionários $\quad(14,8 \%)^{(16,27,29,36)}$, uma 
entrevista $(3,7 \%)^{(19)}$ e dez artigos $(37,0 \%)^{(14,15,17,21-23,27,30,31,37)}$ utilizaram a combinação de dois ou mais instrumentos. A propósito, a observação direta é uma abordagem comum a outros estudos de adesão por ser considerada padrão ouro para monitorização dessa prática $^{(44)}$. Trata-se do único método que proporciona a avaliação referente ao diagnóstico situacional, a necessidade de treinamento e a deficiência da técnica em todos os momentos do cuidado, mesmo havendo possibilidade de mudança de comportamento dos profissionais por serem observados $^{(45)}$.

Os questionários, segundo instrumento mais utilizado nos artigos analisados, possibilitam respostas mais fidedignas por não causarem intimidação aos participantes, como em casos de entrevista na modalidade face a face. Contudo, destaca-se como fragilidade a superestimação da própria aderência à HM criando um viés nos resultados ${ }^{(45)}$.

As publicações que utilizaram a junção de diferentes tipos de instrumentos visaram analisar, além da prática de HM (técnica correta e momentos adequados), a estrutura física, o material disponível para realização da técnica e o nível de conhecimento dos profissionais. Do total de publicações analisadas, apenas três $(11,1 \%)^{(20,21,23)}$ avaliaram também a infraestrutura das instituições, atribuindo a baixa adesão também a fatores institucionais. Nesse contexto, é essencial uma infraestrutura adequada para que se tenha uma prática de HM de forma satisfatória e com os insumos necessários, porém essa realidade está longe do esperado ${ }^{(46)}$.

Citam-se como as principais inadequações referentes à infraestrutura a ausência de lavatório nas enfermarias e a falta de dispensadores de antisséptico. Dessa forma, é fundamental que os gestores apontem as principais fragilidades para criar estratégias que melhorem o ambiente de trabalho, de forma a contemplar os diferentes aspectos que implicam diretamente na baixa adesão às boas práticas de $\mathrm{HM}^{(47)}$.

Verificou-se que 12 artigos $(44,4 \%)^{(15-}$ 17,19-23,25,27,29,36) utilizam questionários ou entrevistas para avaliar o conhecimento e a adesão do profissional acerca da temática. Os achados mais relevantes e de maior frequência apontam a existência de lacunas do conhecimento. Em relação ao treinamento prévio, dos artigos que avaliaram o conhecimento, sete $(25,9 \%)^{(15,21,25,29,31,33,36)}$ apontaram a sua realização.

O cumprimento dos padrões de HM foi analisado, principalmente, mediante a observação $\operatorname{direta}^{(11-13,15,17,18,20-24,26-28,30,32-35,37-}$ ${ }^{40)}$, contendo as etapas das lavagens das mãos, o tempo gasto, situações em que se lavavam as mãos, avaliação na retirada de adornos e tamanho das unhas. Considerava-se como 
adesão insatisfatória a negligência ou a não realização de uma das etapas da técnica preconizada, bem como a inobservância dos fatores que configuram as boas práticas de HM. A adesão da técnica de HM foi mensurada de forma qualitativa.

Os profissionais, participantes de alguns artigos $\operatorname{analisados}^{(12,17-20,26,27)}$, além de executarem a técnica erroneamente, realizavam-na com mais frequência após o contato com o paciente ${ }^{(14,24,26,32,39)}$, deixando de cumprir, corretamente, os cinco momentos da HM. Em um estudo realizado com profissionais de saúde em ambiente hospitalar, revelou-se que a maioria dos profissionais tende a higienizar as mãos com maior frequência antes da realização de procedimento asséptico e após situações de risco de contato com fluidos corporais ${ }^{(48)}$. Esse aspecto reforça a necessidade de avaliação da adesão à técnica nos cinco momentos preconizados pela OMS, em conformidade com os instrumentos utilizados por alguns artigos analisados.

Ademais, existem vários aspectos para a não aderência do profissional a tal prática, entre eles estão aspectos materiais, comportamentais ou institucionais ${ }^{(49)}$. A estratégia multimodal, utilizada como instrumento em alguns artigos analisados, comporta todas essas dimensões, favorecendo a mudança de comportamento, o clima de segurança, alterações na infraestrutura, incentivo na formação dos profissionais, além de avaliações periódicas e o retorno (feedback) das estratégias utilizadas ${ }^{(4)}$.

Vale ressaltar que a confiabilidade dos dados coletados depende tanto da validação do instrumento quanto da qualidade da implementação do processo de coleta de dados. Em relação aos resultados obtidos por este estudo, a minoria dos instrumentos é validada, entretanto utilizar mais de um instrumento pode facilitar a obtenção de dados mais fidedignos que retratem a realidade vivida pelos profissionais ${ }^{(50)}$.

É imperiosa a necessidade de intervenções adequadas a cada realidade institucional visando a mudanças comportamentais e ao desempenho de procedimentos mais eficazes, evitando danos e garantindo a segurança do paciente ${ }^{(42)}$. É imprescindível o uso de instrumentos adequados e validados para a avaliação da HM, garantindo a replicação periódica das avalições com o mesmo grau de confiabilidade.

\section{CONCLUSÃO}

Os achados apontaram a existência de instrumentos validados para avaliar a adesão às boas práticas de HM. A observação não participante foi o método mais utilizado e, em algumas pesquisas, utilizaram-se questionários ou entrevistas em associação com a observação. Majoritariamente, a 
percepção da adesão à prática de $\mathrm{HM}$ foi qualitativa, mediante o cumprimento do passo a passo da técnica preconizada.

Destaca-se como limitação do estudo a falta de padronização metodológica que impossibilita ou dificulta a realização de comparações entre a maior parte dos estudos.

Faz-se necessário o incentivo à aplicação de instrumentos validados por parte das instituições/pesquisadores, principalmente aqueles que se configurem em estratégia multimodal que avaliem, além da técnica, outros fatores, podendo diminuir os vieses nos dados referentes à adesão às boas práticas de HM.

\section{REFERÊNCIAS}

1. Oliveira HM, Silva CPR, Lacerda RA. Políticas de controle e prevenção de infecções relacionadas à assistência à saúde no Brasil: análise conceitual. Rev Esc Enferm USP. [Internet]. 2016; [acesso em: 16 jan. 2021];50(3):505-511. Disponível em: https://www.scielo.br/scielo.php?script=sci_ar ttext\&pid=S0080-

62342016000300505\&lng=en\&tlng=en

2. Paula DG, Pinto FF, Silva RFA, Paula VG. Estratégias de adesão à higienização das mãos por profissionais de saúde. Revista de Epidemiologia e Controle de Infecção [Internet]. 2017 [acesso em: 16 jan. 2021]; 7(2):113-21. Disponível em: https://online.unisc.br/seer/index.php/epidemi ologia/article/view/7731

3. Belela-Anacleto ASC, Peterlini MAS, Pedreira MLG. Higienização das mãos como prática do cuidar: reflexão acerca da responsabilidade profissional. Rev Bras Enferm. [Internet]. 2017 [acesso em: 16 jan. 2021];70(2):461-64. Disponível em: https://www.scielo.br/scielo.php?script=sci_ar ttext\&pid=S0034-

$71672017000200442 \& \operatorname{lng}=$ en $\&$ tlng=en

4. Valim MD, Rocha ILS, Souza TPM, Cruz YA, Bezerra TB, Baggio E. Eficácia da estratégia multimodal para adesão à Higiene das Mãos: revisão integrativa. Rev Bras Enferm [Internet]. 2019 [acesso em: 16 jan. 2021];72(2): 578-92. Disponível em: https://www.scielo.br/pdf/reben/v72n2/pt_003 4-7167-reben-72-02-0552.pdf

5. Boeira ER, Souza ACS, Pereira MS, Vila VSC, Tipple AFV. Controle de infecções e medidas de segurança do paciente abordados em projetos pedagógicos da enfermagem. Rev esc enferm USP [Internet]. 2019 [acesso em: 16 jan. 2021];53:e03420. Disponível em: https://www.scielo.br/scielo.php?script=sci_ar ttext\&pid=S0080-

$62342019000100407 \&$ tlng=en

6. Tartari E, Pires D, Bellissimo RF, De Kraker M, Borzykowski TH, Allegranzi B et al. The global hand-sanitizing relay: promoting hand hygiene through innovation. J Hosp Infect. [Internet] 2017 [acesso em: 17 jan. 2021];95(2):189-93. Available from: https://www.ncbi.nlm.nih.gov/pubmed/28081 910

7. Souza MT, Silva MD, Carvalho RC. Revisão integrativa: o que é e como fazer. Einstein [Internet]. 2010 [acesso em: 17 jan. 2021];8(1):102-6. Disponível em: http://www.scielo.br/pdf/eins/v8n1/pt_16794508-eins-8-1-0102.pdf

8. Mendes KDS, Silveira RCCP, Galvão CM. Use of the bibliographic reference manager in the selection of primary studies in integrative reviews. Texto contexto - enferm [Internet]. 2019 [acesso em: 17 jan. 2021];28:e20170204. Available from: https://www.scielo.br/scielo.php?script=sci_ar ttext\&pid=S0104-

$07072019000100602 \&$ tlng $=$ en 
9. Galvão TF, Pansani TSA, Harrad D. Principais itens para relatar Revisões sistemáticas e Meta-análises: A recomendação PRISMA. Epidemiol Serv Saúde [Internet]. 2015 [acesso 17 jan. 2021];24(2):335-42. Disponível em: https://www.scielo.br/scielo.php?script=sci_ar ttext\&pid=S2237-96222015000200335

10. Cieto BB, Garbuio DC, Camargo VB, Napoleão AA. Nursing resources and innovations for hospital discharge: an integrative review. REME [Internet]. 2014 [acesso em: 17 jan. 2021];18(3):758-63. Available from: http://bases.bireme.br/cgibin/wxislind.exe/iah/online/?IsisScript=iah/ia h. $x$ is $\&$ src $=$ google $\&$ base $=$ BDENF\&lang $=p \& n$ extAction=lnk\&exprSearch=27015\&indexSea $\mathrm{rch}=\mathrm{ID}$

11. São S, Bartoloni A, Virgili G, Mannelli F, Fumagalli S, Di Martino $\mathrm{P}$ et al. Marked variability in adherence to hand hygiene: A 5unit observational study in Tuscany. Am J Infect Control [Internet]. 2009 [acesso em: 01 mar. 2021]; 37(4): 306-10. Available from: https://www.ajicjournal.org/article/S01966553(08)00756-6/fulltext

12. Martinez MR, Campos LAAF, Nogueira PCK. Adesão à técnica de lavagem de mãos em Unidade de Terapia Intensiva Neonatal. Rev paul pediatr [Internet]. 2009 [acesso em: 01 mar. 2021]; 27(2):179-85. Disponível em: https://www.scielo.br/scielo.php?script=sci_ar ttext\&pid=S0103-

05822009000200010\&lng=pt\&tlng=pt

13. Garcia MR, Rodrigo HR, Álvarez FC, Juarez FMA, Núñez MLF, Marta CL et al. Evaluating the impact of a hand hygiene campaignon improving adherence. Am J Infect Control. [Internet]. 2010 [acesso em: 01 mar. 2021];38(3):240-3. Available from: https://www.ajicjournal.org/article/S01966553(09)00896-7/fulltext

14. Korniewicz DM, El-Masri M. Exploring the factors associated with hand hygiene compliance of nurses during routine clinical practice. Appl Nurs Res. [Internet]. 2010 [acesso em: 01 mar. 2021];23(2):86-90. Available from: https://www.sciencedirect.com/science/article /pii/S0897189708000505?via\%3Dihub

15. McLaughlin AC, Walsh F. Individual differences in judgments of hand hygiene risk by health care workers. Am J Infect Control [Internet]. 2011 [acesso em: 01 mar. 2021];39(6):456-63. Available from: https://www.ajicjournal.org/article/S01966553(10)00938-7/fulltext

16. Atti MLCD, Tozzi A, Ciliento G, Pomponi M, Rinaldi S, Raponi M. Health care workers' and parents' perceptions of measures for improving adherence to handhygiene. BMC Public Health [Internet]. 2011 [acesso em: 01 mar. 2021]; 11:466. Available from:

https://bmcpublichealth.biomedcentral.com/tr ack/pdf/10.1186/1471-2458-11-466

17. Cantón CR, Sanmartín BN, Casas LP. Evaluación de la técnica de higiene de manos em profesionales asistenciales. Rev Calid Asist [Internet]. 2011 [acesso em: 01 mar. 2021]; 26(6):376-9. Available from: https://www.sciencedirect.com/science/article /abs/pii/S1134282X11001576?via\%3Dihub

18. Locks L, Lacerda LT; Gomes E, Tine ACPS. Qualidade da higienização das mãos de profissionais atuantes em unidades básicas de saúde. Rev Gaúcha Enferm [Internet]. 2011 [acesso em: 01 mar. 2021]; 32(3):569$75 . \quad$ Disponível em: https://www.scielo.br/scielo.php?script=sci_ar ttext\&pid=S1983$14472011000300019 \& \operatorname{lng}=\mathrm{pt} \& \operatorname{tng}=\mathrm{pt}$

19. Joshi SC, Diwan V, Tamhankar AJ, Joshi $\mathrm{R}$, Shah H, Sharma H et al. Qualitative study on perceptions of hand hygiene among hospital staff in a rural teaching hospital in India. J Hosp Infect [Internet]. 2012 [acesso em: 01 mar. 2021];80(4):340-4. Available from: 
https://www.journalofhospitalinfection.com/a rticle/S0195-6701(12)00016-3/fulltext

20. Silva FM, Porto TP, Rocha PK, Lessmann JC, Cabral PFA, Schneider KLK. Higienização das mãos e a segurança do paciente pediátrico. Cienc Enferm [Internet]. 2013 [acesso em: 01 mar. 2021]; 19(2):99109. Disponível em: ht https://scielo.conicyt.cl/scielo.php?script=sci_ arttext\&pid=S0717-

$95532013000200010 \& \operatorname{lng}=\mathrm{en} \& \mathrm{nrm}=\mathrm{iso} \& \ln \mathrm{ln}$ =entp://dx.doi.org/10.4067/S0717-

95532013000200010

21. Prado MF, Hartmann TPST, Filho LA. Acessibilidade da estrutura física hospitalar para a prática da higienização das mãos. Esc Anna Nery [Internet]. 2013 [acesso em: 01 mar. 2021];17(2):220-6. Disponível em:

https://www.scielo.br/scielo.php?script=sci_ar ttext\&pid=S1414-

$81452013000200003 \& \operatorname{lng}=p t \& \operatorname{lng}=p t$

22. Allegranzi B, Gayet-Ageron A, Damani $\mathrm{N}$, Bengaly L, McLaws ML, Moro ML et al. Global implementation of WHO's multimodal strategy for improvement of hand hygiene: a quasi-experimental study. Lancet Infect Dis [Internet]. 2013 [acesso em: 01 mar. 2021];13(10):843-51. Available from: https://www.thelancet.com/action/showPdf?pi $\mathrm{i}=\mathrm{S} 1473-3099 \% 2813 \% 2970163-4$

23. Bathke J, Cunico PA, Maziero ECS, Cauduro FLF, Sarquis LMM, Cruz EDA. Infraestrutura e adesão à higienização das mãos: desafios à segurança do paciente. Rev Gaúcha Enferm [internet]. 2013 [acesso em: 01 mar. 2021];34(2):78-85. Disponível em: https://www.scielo.br/scielo.php?script=sci_ar ttext\&pid=S1983-

$14472013000200010 \& \operatorname{lng}=p t \& \operatorname{lng}=p t$

24. Uneke JC, Ndukwe CD, Oyibo PG, Nwakpu KO, Nnabu RC, Prasopa-Plaizier N. Promotion of hand hygiene strengthening initiative in a Nigerian teaching hospital: implication for improved patient safety in low-income health facilities. Braz J Infect Dis [Internet]. 2013 [acesso em: 01 mar. 2021]; 18(1):21-7. Available from: https://reader.elsevier.com/reader/sd/pii/S141 3867013001943?token=D85E9E6EF4332013 E99B93DD1EA47F95356EF378C4355D655 CCF115717E4B46D8CF4222434CF9402DA 69099F75186FB8

25. Pérez $\mathrm{P}$, Usagre $\mathrm{MH}$, Cavanilla $\mathrm{AB}$, Humada MAS, Camacho 5 BB, Vázquez M. Higiene de las manos: conocimientos de los profesionales y áreas de mejora. Cad Saúde Pública [Internet]. 2015 [acesso em: 01 mar. 2021];31(1):149-60. Available from: https://www.scielo.br/scielo.php?script=sci_ar ttext\&pid=S0102-

$311 \mathrm{X} 2015000100149 \& \operatorname{lng}=\mathrm{es} \& \operatorname{tn} g=\mathrm{es}$

26. Souza LM, Ramos MF, Becker ES, Meirelles LCS, Monteiro SAP. Adesão dos profissionais de terapia intensiva aos cinco momentos da higienização das mãos. Rev Gaúcha Enferm [Internet]. 2015 [acesso em: 01 mar. 2021]; 36(4):21-28. Disponível em: https://www.scielo.br/scielo.php?script=sci_ar ttext\&pid=S1983-

$14472015000400021 \& \operatorname{lng}=$ en $\&$ tlng=en

27. Sandoval LJS, Boulangger JFB, García DSC. Adherencia al lavado de manos en personal de salud del hospital regional José Alfredo Mendoza Olavarria II-2 de Tumbes. Rev iberoam Educar Investi Doente [internet]. 2016 [acesso em: 01 mar. 2021]; 6(4):42-54. Available from: https://www.enfermeria21.com/revistas/aladef e/articulo/221/

28. Dufour JC, Reynier P, Boudjema S, Soto Aladro Um, Giorgi R, Brouqui P. Evaluation of hand hygiene compliance and associated factors with a radio-frequency-identificationbased real-time continuous automated monitoring system. J Hosp Infect [Internet]. 2017 [acesso em: 01 mar. 2021]; 95(4):33451. Available from: https://www.journalofhospitalinfection.com/a rticle/S0195-6701(17)30063-4/fulltext 
29. Oliveira AC, Paula AO. A percepção dos profissionais de saúde em relação à higienização das mãos. Rev Fund Care Online [internet]. 2017 [acesso em: 01 mar. 2021];9(2)321-6. Disponível em: http://seer.unirio.br/index.php/cuidadofundam ental/article/view/3832

30. Nobile, M, Conti C, Bastianelli A, Piscitelli A, Calori GM, Navone P. Promotion of hand hygiene: the experience of the orthopaedic hospital Gaetano Pini-CTO, Milan, Ital. Ann Ig [internet]. 2018 [acesso em: 01 mar. 2021] 30(3):229-36. Available from:

https://www.researchgate.net/publication/324 867138_Promotion_of_hand_hygiene_the_ex perience_of_the_orthopaedic_hospital_Gaeta no_Pini-CTO_Milan_Italy

31. Phan HT, Tran HTT, Tran HTM, Dinh APP, Ngo HT, Haglow JT, et al. An educational intervention to improve hand hygiene compliance in Vietnam. BMC Infect Dis [internet]. 2018 [acesso em: 01 mar. 2021]; 18:116. Available from: https://bmcinfectdis.biomedcentral.com/articl es/10.1186/s12879-018-3029-5

32. Luciano MNF, Nascimento BB, Nunes EM, Oliveira LFM, Davim RMB, Alves ESRC.

Adherence to hand hygienize by health profes sionals in an Intensive Care Unit. J Nurs UFPE on line [internet]. 2017 [acesso em: 01 mar. 2021]; 11(10):3764-70. Available from: https://periodicos.ufpe.br/revistas/revistaenfer magem/article/view/22672/24280

33. Oliveira AC, Gama CS, Paula AO. Multimodal strategy to improve the adherence to hand hygiene and self-assessment of the institution for the promotion and practice of hand hygiene. Journal of Public Health [internet] 2018 [acesso em 01 mar. 2021]; 40(1):163-8. Available from: https://academic.oup.com/jpubhealth/article/4 0/1/163/3077005
34. LLAPA-RODRÍGUE, E.O. Aderência de profissionais de saúde à higienização das mãos. Rev enferm UFPE on line [Internet]. 2018 [acesso em 01 mar. 2021]; 12(6):157885. Disponível em: < https://periodicos.ufpe.br/revistas/revistaenfer magem/article/view/230841

35. Dijk MDV, Mulder SA, Erasmus V, Beeck AHEV, Vermeeren JMJJ, Liu X, et al. A multimodal regional intervention strategy framed as friendly competition to improve hand hygiene compliance. Infection Control \& Hospital Epidemiology [Internet]. 2019 [acesso em 01 mar. 2021]; 20(2):187-93. Available from: https://www.cambridge.org/core/journals/infe ction-control-and-hospitalepidemiology/article/multimodal-regionalintervention-strategy-framed-as-friendlycompetition-to-improve-hand-hygienecompliance/18D993BFB0B3502E707271A69 DE21842

36. Lai C, Lu M, Tang H, Chen Y, Wu Y, Chiang $\mathrm{H}$, et al. Implementation of a national quality improvement program to enhance hand hygiene in nursing homes in taiwan. Journal of Microbiology, Immunology and infection [Internet] 2019 [acesso em 01 mar. 2021]; 52(2):345-51. Available from: https://www.sciencedirect.com/science/article /pii/S1684118218304122?via\%3Dihub

37. Leite MAP, Oliveira MA, Leuthier RM, Filho JRO, Fernandes LGA, Santos AF, et al. Higienização das mãos: conhecimentos e atitudes de profissionais da saúde. Rev enferm UFPE on line [Internet] 2019 [acesso em: 01 mar. 2021]; 13:e236418. Disponível em: <https://periodicos.ufpe.br/revistas/revistaenf ermagem/article/view/236418/32758

38. Fouad, M; Eltaher, S. Hand hygiene initiative: comparative study of pre-and postintervention outcomes. EMHJ [Internet] 2020. [acesso em: 01 mar. 2021] 26(2):198205. Available from: <https://doi.org/ 10.26719/2020.26.2.198 
39. Paula, DG et al. A higienização das mãos em setores de alta complexidade como elemento integrador no combate ao SarsCoV-2.Rev. Bras. Enferm. [Internet] 2020 [acesso em: 01 mar. 2021] 73:e20200316. Disponível em: $<$ http://dx.doi.org/10.1590/0034-7167-20200316

40. Zhong, $X$ et al. Comparison of two electronic hand hygiene monitoring systems in promoting hand hygiene of healthcare workers in the intensive care unit. BMC Infectious Diseases. [Internet] 2021 [acesso em: 01 mar. 2021]: 21(50). Available from: https://doi.org/10.1186/s12879-020-05748-3

41. Colaço C, Sousa PP. Ashesion to hygiene of hands: a nursing research. Rev UNINGÁ Review [Internet] 2017 [acesso em: 3 mar. 2021]; 30(1)11-14. Available from: https://www.mastereditora.com.br/periodico/2 0170401_235052.pdf

42. Graveto JMGN, Rebola RIF, Fernandes EA, Costa PJS. Higiene das mãos - adesão dos enfermeiros após processo formativo. Rev Bras Enferm [Internet]. 2018 [acesso em: 3 mar. 2021];71(3):1189-93. Disponível em: https://www.scielo.br/scielo.php?script=s ci_arttext\&pid=S0034-

$71672018000301189 \& \operatorname{lng}=\mathrm{en} \& \operatorname{tln} \mathrm{g}=\mathrm{en}$

43. Souza LMB, Alievi MF, Piasentin CZ, Bandeira VAC, Loro MM, Stumm EMF et al. Análise do conhecimento dos profissionais de enfermagem em relação à higienização das mãos. R Epidemiol Control Infec [Internet]. 2018 [acesso em: 3 mar. 2021];8(2);142-9. Disponível em: https://online.unisc.br/seer/index.php/epidemi ologia/article/view/11199

44. Janotti L, Junior WVM. Adequação de um instrumento de monitoramento de higienização das mãos de um hospital do Rio de Janeiro. $\mathrm{R}$ Epidemiol Control Infec [Internet]. 2018. [acesso em 3 mar. 2021]; 8(3):195-203. Disponível em: https://online.unisc.br/seer/index.php/epidemi ologia/article/view/10765

45. Oliveira Ac, Paula AO, Gama CS. Monitorização da higienização das mãos: observação direta versus taxa autorreportada. Enfermería Global [Internet]. 2017 [acesso: em 3 mar. 2021]; 16(4);324-53. Disponível em:

https://revistas.um.es/eglobal/article/view/277 $861 / 217141$

46. Moura PMM, Tristão FSA, Guanilo MEE, Porto, AR. Avaliação da infraestrutura hospitalar para a higienização das mãos. Rev enferm UFPE online [Internet]. 2017 [acesso em: 3 mar. 2021];11Supl. 12:5289-96. Disponível em: https://periodicos.ufpe.br/revistas/revistaenfer magem/article/view/22884/25480

47. Vasconcelos RO, Alves DCI, Fernandes LM, Oliveira JLC. Adhesión a la higiene de las manos por el equipo de enfermería en la unidad de cuidados intensivos. Rev enfermería global [Internet]. 2018 [acesso em: 3 mar. 2021]; 17(50):430-45. Disponível em: https://revistas.um.es/eglobal/article/view/284 131

48. Araujo AP, Nóbrega GB, Santos LFC, Aragão RSA, Pontes AAN. Análise da higienização das mãos pelos profissionais de saúde em ambiente hospitalar durante dois meses. Revista Saude e Ciencia online [Internet]. 2015 [acesso em: 3 mar. 2021]; 4(3):44-54. Disponível em: https://pdfs.semanticscholar.org/4cba/9ac243 3539281dd80a87bf528ce090092ef5.pdf

49. Dourado SBPB. Hand hygiene: its effects on infection índices and hospital costs. Rev enferm UFPE on line [Internet]. 2016 [acesso em: 3 mar. 2021]; 10Supl. 4:S3585-92. Available from: https://periodicos.ufpe.br/revistas/revistaenfer magem/article/view/11133/12627

50. Oliveira AC, Paula AO, Gama CS, Oliveira JR, Rodrigues CD. Adesão à 
higienização das mãos entre técnicos de enfermagem em um hospital universitário. Rev enferm UERJ [Internet]. 2016 [acesso em: 3 mar. 2021];24(2):1-6. Disponível em: https://www.e- publicacoes.uerj.br/index.php/enfermagemuer j/article/view/9945

Submissão: 2021-03-21

Aprovado: 2021-05-19 